\title{
Opioid-Free Total Knee Arthroplasty? Local Infiltration Analgesia Plus Multimodal Blood-Loss Prevention Make it Possible
}

\author{
E. Carlos Rodriguez-Merchan, MD, PhD • Alfonso Vaquero-Picado, MD, FEBOT • Juan S. Ruiz-Perez, MD
}

Received: 30 June 2018/Accepted: 13 September 2018/Published online: 15 October 2018

(C) Hospital for Special Surgery 2018

\begin{abstract}
Opioids have been widely used in the USA for pain control after total knee arthroplasty (TKA). However, adverse effects, especially the possibility of addiction, have increased interest in opioid-free pain management after surgery. We therefore sought to review current pain management protocols after TKA, focusing especially on opioid-free alternatives. We reviewed the literature on pain management after TKA using Medline (PubMed), through June 30, 2018, using the keywords "TKA" and "analgesia." We found 388 articles but chose to analyze the 34 that presented high-quality (levels I and II) evidence. Local infiltration analgesia (LIA) is a good option for reducing the use of post-operative opioids; many reports have compared LIA against a nerve block or studied the synergies between two protocols of loco-regional anesthesia. Multimodal blood-loss prevention is sometimes recommended in combination with opioid-free analgesia. In most studies, however, no differences are reported or contradictory results exist. Post-operative pain management protocols vary so much that it is difficult to strongly favor a determined pathway.
\end{abstract}

Keywords knee total knee arthroplasty. post-operative pain - opioids - local infiltration analgesia . opioid-free TKA

Total knee arthroplasty (TKA) is one of the most frequently performed orthopedic procedures. In the USA, the rate of TKA is $235 / 100,000$ population [7], representing about $9 \%$ of total inpatient stays.

Electronic supplementary material The online version of this article (https://doi.org/10.1007/s11420-018-9636-2) contains supplementary material, which is available to authorized users.

E. Carlos Rodriguez-Merchan, $\mathrm{MD}, \mathrm{PhD}(\triangle)$ •

A. Vaquero-Picado, MD, FEBOT · J. S. Ruiz-Perez, MD

Department of Orthopedic Surgery, Knee Surgery Unit,

La Paz University Hospital_-IdiPaz,

Paseo de la Castellana 261,

28046, Madrid, Spain

e-mail: ecrmerchan@hotmail.com
Several advances have been developed to facilitate early discharge after TKA, and many hospitals have implemented "fast-track" protocols. While strategies focused on reducing intra- and post-operative bleeding facilitate patient recovery and diminish the rate of post-operative transfusions [25], improvements in post-operative pain management during the last decade, including multimodal interventions, have also facilitated TKA recovery. Combined anesthesia protocols (i.e., general or epidural anesthesia plus peripheral nerve blocks), regional anesthesia (i.e., intradural), and local infiltration analgesia (LIA) protocols have provided popular methods for decreasing pain and accelerating recovery after TKA. Nonetheless, opioids have been widely prescribed in the USA for control of knee pain both before and after TKA [8, 14, 19]. Consequently, adverse effects, especially the possibility of dependence and addiction associated with their use, have increased interest in non-opioid post-surgical pain management strategies [14, 19].

We decided to review the literature on pain control after TKA, especially regarding non-opioid alternatives. Is it possible to adequately manage pain after TKA without opioids? Is the LIA approach a valid alternative?

We searched Medline (PubMed) using the keywords "TKA" (total knee arthroplasty) and "analgesia," through June 30, 2018. We retrieved 388 articles but analyzed only the 34 that provided grades I and II levels of evidence.

Almost all the studies retrieved reported that LIA can provide good pain control in the first $48 \mathrm{~h}$ after TKA. It usually is utilized as a part of a multimodal approach for post-operative pain management $[9,30,31]$. Different compositions of LIA have been used. Most studies include a local anesthetic (usually ropivacaine) combined with a corticosteroid (usually betamethasone) $[2,21]$. Morphine, epinephrine, and antibiotics can also be added, but there is little evidence of their effectiveness [29]. 
LIA is typically employed to reduce post-operative opioid use $[4,11,16,22]$. Many studies have compared LIA against a nerve block or have examined combined local and regional anesthesia protocols $[3,5,10,12,13,20,27,32$ 34]. Most show no differences, and contradictory results between studies exist. Post-operative pain management protocols vary so much that it is difficult to present evidence favoring a particular pathway.

LIA seems to be a good alternative to facilitate early rehabilitation on "fast-track" protocols, as its control of pain is especially important during the first $48 \mathrm{~h}[1,5,21,22,28]$. Although differences between protocols at mid- to long-term follow-up have not been well-documented, LIA seems not to increase complications with wound healing or infection [15, 18]. LIA has been demonstrated to be a safe and useful alternative for ensuring early pain control, for avoiding motor blocks, and for reducing opioid usage. No major complications in the post-operative period have been found, although long-term functional outcomes should be studied.

In our surgical practice, we have used a single-dose LIA approach for almost 8 years [6, 17, 23-25]. Our cocktail consists of $50 \mathrm{cc}$ of saline, epinephrine $300 \mu \mathrm{g}$, morphine sulfate $10 \mathrm{mg}$, tobramycin $100 \mathrm{mg}$, betamethasone sodium phosphate $6 \mathrm{mg}$, betamethasone acetate $6 \mathrm{mg}$, and ropivacaine $200 \mathrm{mg}$. We inject the total volume of $80 \mathrm{cc}$ into the posterior capsule, collateral ligaments, pes anserinus, and patellar and quadriceps tendons after washing the bone surfaces and before definitive implantation.

All surgeries are performed using epidural anesthesia and a multimodal blood-loss prevention approach (MBLPA). MBLPA includes preoperative hemoglobin evaluation and optimization with intravenous iron in patients whose levels are under $13 \mathrm{~g} / \mathrm{dL}$. Hypotensive anesthesia and a tourniquet at $100 \mathrm{mmHg}$ above the systolic pressure for the whole procedure are also included. Several technical tips, including plugging the femoral canal with autologous bone graft obtained from the femoral cuts, avoiding drains, and releasing the tourniquet after wound closure, are also used to minimize blood loss. Finally, tranexamic acid (TXA) has been used in two different protocols. One protocol includes intravenous TXA administered in two doses of $15 \mathrm{mg} / \mathrm{kg}$ in saline $100 \mathrm{cc} 15$ to $20 \mathrm{~min}$ before tourniquet release and $3 \mathrm{~h}$ later. The other protocol (and mostly used at this moment) includes intra-articular TXA administration ( $2 \mathrm{~g}$ in $20 \mathrm{cc}$ of saline) after wound closure, without intravenous doses. Good results have been reported with such protocols in primary TKA, revision TKA, and hemophilic patients $[17,24-26]$.

Patients can move the knee from the first day and are usually discharged 2 days post-surgery walking with crutches. We use low-molecular-weight heparin (LMWH) for 4 weeks for thromboembolic prophylaxis. No opioids are used after the first day, and patients are discharged with oral analgesics (usually acetaminophen and nonsteroidal anti-inflammatory medications).

In conclusion, although opioids have been widely used for pain control following TKA, multiple level I and II studies have been published in recent years supporting the use of LIA and MBLPA for improving pain control and accelerating recovery after TKA.

\section{Compliance with Ethical Standards}

Conflict of Interest: E. Carlos Rodriguez-Merchan, MD, PhD, Alfonso Vaquero-Picado, MD, FEBOT, and Juan S. Ruiz-Perez, MD, declare that they have no conflicts of interest.

\section{Human/Animal Rights: N/A}

\section{Informed Consent: N/A}

Required Author Forms: Disclosure forms provided by the authors are available with the online version of this article.

\section{References}

1. Barastegui D, Robert I, Palau E, Haddad S, Reverte-Vinaixa M, Lorente L, et al. Can local infiltration analgesia increase satisfaction in post-operative short-term pain control in total knee arthroplasty? J Orthop Surg Hong Kong. 2017; 25:2309499017690461.

2. Breindahl T, Simonsen O, Hindersson P, Brødsgaard Dencker B, Brouw Jørgensen M, Rasmussen S. Autologous blood transfusion after local infiltration analgesia with ropivacaine in total knee and hip arthroplasty. Anesthesiol Res Pract. 2012;2012:458795.

3. Chaubey D, Mahajan HK, Chauhan PR, Govind PS, Singh P, Dhanevar R, et al. Comparison of continuous femoral nerve block versus local infiltration analgesia as a postoperative analgesia in unilateral total knee arthroplasty. J Clin Diagn Res. 2017;11:UC13-UC16.

4. Essving P, Axelsson K, Kjellberg J, Wallgren O, Gupta A, Lundin A. Reduced morphine consumption and pain intensity with local infiltration analgesia (LIA) following total knee arthroplasty. Acta Orthop. 2010;81:354-360.

5. Fan L, Yu X, Zan P, Liu J, Ji T, Li G. Comparison of local infiltration analgesia with femoral nerve block for total knee arthroplasty: a prospective, randomized clinical trial. $J$ Arthroplasty. 2016;31:1361-1365.

6. Gomez-Barrena E, Ortega-Andreu M, Padilla-Eguiluz NG, Pérez-Chrzanowska H, Figueredo-Zalve R. Topical intraarticular compared with intravenous tranexamic acid to reduce blood loss in primary total knee replacement: a double-blind, randomized, controlled, noninferiority clinical trial. J Bone Joint Surg Am. 2014;96:1937-1944.

7. Inacio MCS, Paxton EW, Graves SE, Namba RS, Nemes S. Projected increase in total knee arthroplasty in the United States - an alternative projection model. Osteoarthritis Cartilage. 2017;25:1797-1803.

8. Inacio MCS, Cashman K, Pratt NL, Gillam MH, Caughey G, Graves SE, et al. Prevalence and changes in analgesic medication utilisation 1 year prior to total joint replacement in an older cohort of patients. Osteoarthritis Cartilage. 2018;26:356-362.

9. Kampitak W, Tanavalee A, Ngarmukos S, Amarase C, Apihansakorn R, Vorapalux P. Does adductor canal block have a synergistic effect with local infiltration analgesia for enhancing ambulation and improving analgesia after total knee arthroplasty? Knee Surg Relat Res. 2018;30:133-141.

10. Kampitak W, Tanavalee A, Ngarmukos S, Amarase C, Songthamwat B, Boonshua A. Comparison of adductor canal block versus local infiltration analgesia on postoperative pain and functional outcome after total knee arthroplasty: a randomized controlled trial. Malays Orthop J. 2018;12:7-14. 
11. Keijsers R, van Delft R, van den Bekerom MPJ, de Vries DC, Brohet RM, Nolte PA. Local infiltration analgesia following total knee arthroplasty: effect on post-operative pain and opioid consumption-a meta-analysis. Knee Surg Sports Traumatol Arthrosc. 2015;23:1956-1963.

12. Li J, Deng X, Jiang T. Combined femoral and sciatic nerve block versus femoral and local infiltration anesthesia for pain control after total knee arthroplasty: a meta-analysis of randomized controlled trials. J Orthop Surg. 2016;11:158.

13. Ma L-P, Qi Y-M, Zhao D-X. Comparison of local infiltration analgesia and sciatic nerve block for pain control after total knee arthroplasty: a systematic review and meta-analysis. J Orthop Surg. 2017;12:85.

14. Madras BK. The president's commission on combating drug addiction and the opioid crisis: origins and recommendations. Clin Pharmacol Ther. 2018 23. https://doi.org/10.1002/cpt.1050

15. Milani P, Castelli P, Sola M, Invernizzi M, Massazza G, Cisari C. Multimodal analgesia in total knee arthroplasty: a randomized, double-blind, controlled trial on additional efficacy of periarticular anesthesia. J Arthroplasty 2015;30:2038-2042.

16. Mont MA, Beaver WB, Dysart SH, Barrington JW, Del Gaizo DJ. Local infiltration analgesia with liposomal bupivacaine improves pain scores and reduces opioid use after total knee arthroplasty: results of a randomized controlled trial. $J$ Arthroplasty. 2018;33:90-96.

17. Moráis S, Ortega-Andreu M, Rodríguez-Merchán EC, Padilla Eguiluz NG, Pérez-Chrzanowska H, Figueredo-Zalve R, GómezBarrena E. Blood transfusion after primary total knee arthroplasty can be significantly minimised through a multimodal blood-loss prevention approach. Int Orthop. 2014;38:347-354.

18. Mulford JS, Watson A, Broe D, Solomon M, Loefler A, Harris I. Short-term outcomes of local infiltration anaesthetic in total knee arthroplasty: a randomized controlled double-blinded controlled trial. ANZ J Surg. 2016;86:152-156.

19. Murphy DL, Lebin JA, Severtson SG, Olsen HA, Dasgupta N, Dart RC. Comparative rates of mortality and serious adverse effects among commonly prescribed opioid analgesics. Drug Saf. 2018. https://doi.org/10.1007/s40264-018-0660-4

20. Nagafuchi M, Sato T, Sakuma T, Uematsu A, Hayashi H, Tanikawa H, et al. Femoral nerve block-sciatic nerve block vs. femoral nerve block-local infiltration analgesia for total knee arthroplasty: a randomized controlled trial. $B M C$ Anesthesiol. 2015;15:182.

21. Nakagawa S, Arai Y, Inoue H, Kan H, Hino M, Ichimaru S, et al. Comparative effects of periarticular multimodal drug injection and single-shot femoral nerve block on pain following total knee arthroplasty and factors influencing their effectiveness. Knee Surg Relat Res. 2016;28:233-238.

22. Niemelainen M, Kalliovalkama J, Aho AJ, Moilanen T, Eskelinen A. Single periarticular local infiltration analgesia reduces opiate consumption until 48 hours after total knee arthroplasty. A randomized placebo-controlled trial involving 56 patients. Acta Orthop. 2014;85:614-9.
23. Ortega-Andreu M, Pérez-Chrzanowska H, Figueredo R, GómezBarrena E. Blood loss control with two doses of tranexamic acid in a multimodal protocol for total knee arthroplasty. Open Orthop J. 2011;5:44-48.

24. Ortega-Andreu M, Talavera G, Padilla-Eguiluz NG, Perez Chrzanowska H, Figueredo-Galve R, Rodriguez-Merchán, et al. Tranexamic acid in a multimodal blood loss prevention protocol to decrease blood loss in revision total knee arthroplasty: a cohort study. Open Orthop J. 2016;10:439-447.

25. Rodriguez-Merchan EC, Ortega-Andreu M, Padilla-Eguiluz NG, Gomez-Cardero P, Martinez-Lloreda Á, Gomez-Barrena E. Lowvolume formulation of intra-articular tranexamic acid, $25-\mathrm{ml}$ tranexamic acid $(2.5 \mathrm{~g})$ plus $20-\mathrm{ml}$ saline, is effective in decreasing blood transfusion rate in primary total knee replacement even without preoperative haemoglobin optimization. Blood Coagul Fibrinolysis. 2016;27:660-666.

26. Rodriguez-Merchan EC, Romero-Garrido JA, Gomez-Cardero P. Multimodal blood loss prevention approach including intra-articular tranexamic acid in primary total knee arthroplasty for patients with severe haemophilia A. Haemophilia. 2016;22:e318-320.

27. Runge C, Jensen JM, Clemmesen L, Knudsen HB, Holm C, Børglum J, et al. Analgesia of combined femoral triangle and obturator nerve blockade is superior to local infiltration analgesia after total knee arthroplasty with high-dose intravenous dexamethasone. Reg Anesth Pain Med. 2018;43:352-356.

28. Seangleulur A, Vanasbodeekul P, Prapaitrakool S, et al. The efficacy of local infiltration analgesia in the early postoperative period after total knee arthroplasty: a systematic review and meta-analysis. Eur J Anaesthesiol. 2016;33:816-831.

29. Schotanus MGM, Bemelmans YFL, van der Kuy PHM, Jansen J, Kort NP. No advantage of adrenaline in the local infiltration analgesia mixture during total knee arthroplasty. Knee Surg Sports Traumatol Arthrosc. 2017;25:2778-2783.

30. Tran J, Schwarzkopf R. Local infiltration anesthesia with steroids in total knee arthroplasty: a systematic review of randomized control trials. J Orthop. 2015;12:S44-50.

31. Tziona D, Papaioannou M, Mela A, Potamianou S, Makris A. Local infiltration analgesia combined with a standardized multimodal approach including an adductor canal block in total knee arthroplasty: a prospective randomized, placebo-controlled, double-blinded clinical trial. J Anesth. 2018;32:326-332.

32. Zhang Z, Yang Q, Xin W, Zhang Y. Comparison of local infiltration analgesia and sciatic nerve block as an adjunct to femoral nerve block for pain control after total knee arthroplasty: a systematic review and meta-analysis. Medicine (Baltimore). 2017;96:e6829.

33. Zhang L-K, Ma J-X, Kuang M-J, Ma XL. Comparison of periarticular local infiltration analgesia with femoral nerve block for total knee arthroplasty: a meta-analysis of randomized controlled trials. J Arthroplasty. 2018;33:1972-8.e4.

34. Zhou M, Ding H, Ke J. Adductor canal block in combination with posterior capsular infiltration on the pain control after TKA. Ir J Med Sci. 2018;187:465-471. 